STRUCTURAL BIOLOGY COMMUNICATIONS

ISSN 2053-230X

\title{
Structure of proliferating cell nuclear antigen (PCNA) bound to an APIM peptide reveals the universality of PCNA interaction
}

\author{
Kodai Hara, ${ }^{a}$ Masayuki Uchida, ${ }^{a}$ Risa Tagata, ${ }^{a}$ Hideshi Yokoyama, ${ }^{\mathrm{a}, \mathrm{b}}$ Yoshinobu \\ Ishikawa, ${ }^{a}$ Asami Hishiki ${ }^{a}$ and Hiroshi Hashimoto ${ }^{a *}$
}

Received 6 January 2018

Accepted 25 February 2018

Edited by A. Nakagawa, Osaka University, Japan

Keywords: proliferating cell nuclear antigen; PCNA; protein-protein interactions; APIM; PIPM.

PDB reference: human PCNA bound to an APIM peptide, $5 y d 8$

Supporting information: this article has supporting information at journals.iucr.org/f

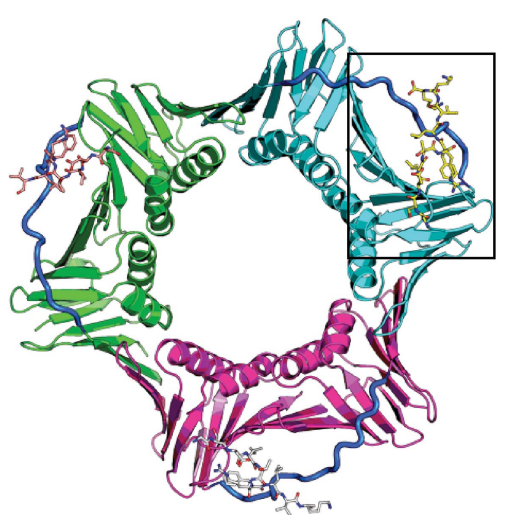

C 2018 International Union of Crystallography

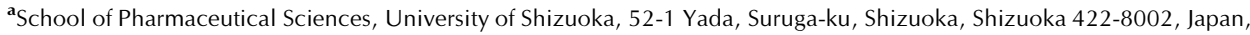
and ${ }^{\mathbf{b}}$ Faculty of Pharmaceutical Sciences, Tokyo University of Science, 2641 Yamazaki, Noda, Chiba 278-8510, Japan. *Correspondence e-mail: hash@u-shizuoka-ken.ac.jp
}

Proliferating cell nuclear antigen (PCNA) provides a molecular platform for numerous protein-protein interactions in DNA metabolism. A large number of proteins associated with PCNA have a well characterized sequence termed the PCNA-interacting protein box motif (PIPM). Another PCNA-interacting sequence termed the AlkB homologue 2 PCNA-interacting motif (APIM), comprising the five consensus residues (K/R)-(F/Y/W)-(L/I/V/A)-(L/I/V/A)$(\mathrm{K} / \mathrm{R})$, has also been identified in various proteins. In contrast to that with PIPM, the PCNA-APIM interaction is less well understood. Here, the crystal structure of PCNA bound to a peptide carrying an APIM consensus sequence, RFLVK, was determined and structure-based interaction analysis was performed. The APIM peptide binds to the PIPM-binding pocket on PCNA in a similar way to PIPM. The phenylalanine and leucine residues within the APIM consensus sequence and a hydrophobic residue that precedes the APIM consensus sequence are crucially involved in interactions with the hydrophobic pocket of PCNA. This interaction is essential for overall binding. These results provide a structural basis for regulation of the PCNA interaction and might aid in the development of specific inhibitors of this interaction.

\section{Introduction}

Proliferating cell nuclear antigen (PCNA) forms a ring-shaped homotrimer and binds double-stranded DNA within the central pore. In combination with its DNA-binding function, PCNA is now known as a bona fide hub protein that physically interacts with hundreds of proteins involved in various nuclear functions, including DNA replication, multiple DNA-repair systems, DNA recombination and sister-chromatid cohesion, and that provides a molecular platform to stimulate the functions of partner proteins (Moldovan et al., 2007). The PCNA-interacting protein box motif, designated PIPM in this work, is known to be a general motif for binding to PCNA (Warbrick et al., 1995; Warbrick, 1998). The consensus sequence of the canonical PIPM is defined as $\mathrm{Q}-X X-(\mathrm{L} / \mathrm{I} / \mathrm{M})-X X-(\mathrm{F} / \mathrm{Y})-$ $(\mathrm{F} / \mathrm{Y})$, where $X$ is any residue (Fig. $1 a)$. In addition to the canonical PIPM, several variants or 'noncanonical PIPMs' have been reported in Y-family DNA polymerases (Pol- $\eta$, Pol- $\iota$ and Pol- $\kappa$; Hishiki et al., 2009). In these noncanonical PIPMs, the glutamine residue at $\mathrm{P} 1$ or the aromatic residue at $\mathrm{P} 8$ is replaced by another amino-acid residue (Fig. 1a).

Independently of PIPM, the AlkB homologue 2 PCNAinteracting motif (APIM) has been identified as a novel PCNA-interacting motif in various proteins (Gilljam et al., 2009). APIM comprises five amino-acid residues, (K/R)(F/Y/W)-(L/I/V/A)-(L/I/V/A)-(K/R), and its sequence 
Table 1

Macromolecule-production information.

\begin{tabular}{ll}
\hline Source organism & H. sapiens \\
Cloning vector & pT7 \\
Expression host & E. coli BL21-CodonPlus(DE3)-RIL \\
Complete amino-acid sequence & MFEARLVQGSILKKVLEALKDLINEACWDI \\
& SSSGVNLQSMDSSHVSLVQLTLRSEGFD \\
& TYRCDRNLAMGVNLTSMSKILKCAGNED \\
& IITLRAEDNADTLALVFEAPNQEKVSDY \\
& EMKLMDLDVELGI PEQEYSCVVKMPS \\
& EFARICRDLSHIGDAVVISCAKDGVKFS \\
ASGELGNGNIKLSQTSNVDKEEEAVTIE \\
MNEPVQLTFALRYLNFFTKATPLSSTVT \\
LSMSADVPLVVEKIADMGHLKYYAPK \\
IEDEEGS \\
\hline
\end{tabular}

conservation is lower than that of PIPM. The expression of an APIM peptide increases the cellular sensitivity to several cytostatic agents, suggesting that APIM is likely to mediate PCNA binding in many proteins involved in DNA repair and cell-cycle regulation during genotoxic stress (Gilljam et al., 2009). To date, many structures of human PCNA bound to PIPMs have been determined and the molecular mechanism

\begin{tabular}{|c|c|c|}
\hline Protein & Residues & \\
\hline ZRANB3 & $1069-1078$ & GSDITRFLVR \\
\hline $\mathrm{ABH} 2$ & $1-7$ & MDRFLVK \\
\hline TFIIS-L & $1-7$ & MDKFVIR \\
\hline TFII-I $\gamma$ APIM1 & $425-434$ & AKERI|RFVIK \\
\hline APIM2 & $530-539$ & VGNRI KFVIK \\
\hline APIM3 & $635-644$ & GSNKI|KFVVK \\
\hline APIM4 & $797-806$ & NKAKI|KFIIK| \\
\hline Topo II $\alpha$ & $962-971$ & TDTTVKFVVK \\
\hline \multirow[t]{3}{*}{ RAD51B } & $228-237$ & LKERN KFLAR \\
\hline & & APIM \\
\hline & & Position \\
\hline Protein & Residues & 12345678910 \\
\hline p21 & $144-164$ & QTSMTDFYHSKRRLIFSKRK P * \\
\hline Pol- $\eta$ & $701-713$ & MQTLESFEPLTH* \\
\hline Pol-ı & $446-458$ & KGLIDYYIMPSLS \\
\hline \multirow[t]{2}{*}{ Pol-к } & $862-870$ & KHTLDIF E $\mathrm{F}$ * \\
\hline & & (a) PIPM \\
\hline
\end{tabular}

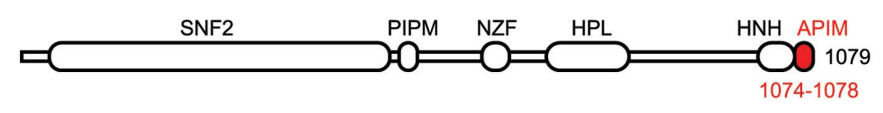

(b)

1069-GSDITRFLVKK-1079

Figure 1

(a) Amino-acid sequences of APIM and PIPM. An asterisk indicates the $\mathrm{C}$-terminus. Numbers above the PIPM sequences indicate the positions of the amino acids in the motif. Positions crucial for interaction with PCNA are shown in red. Note that the residue numbering of Pol- $\iota$ refers to the UniProtKB sequence (Q9UNA4) and differs from that used in a previous structural study, where the N-terminal 25 residues were missing (Hishiki et al., 2009). (b) Domain architecture of human ZRANB3. Human ZRANB3 consists of an SNF2 helicase domain, PIPM, an NZF ubiquitinbinding domain, an HPL (HARP-like) domain, an HNH nuclease motif and APIM. Both PCNA-interacting motifs, PIPM and APIM, make significant contribution to PCNA-dependent recruitment of ZRANB3 to DNA-damage sites, whereas the functional difference between the PIPM and APIM of ZRANB3 in the recruitment is unclarified. The peptide sequence of the human ZRANB3 APIM used in this crystallographic study is shown at the bottom of the domain architecture and the consensus APIM residues are shown in red. underlying PCNA-PIPM interactions has been well characterized, whereas the interaction between PCNA and APIM is less well understood.

Here, we present the crystal structure of human PCNA in complex with an APIM peptide from human ZRANB3, an SWI/SNF helicase involved in the template-switching pathway to restart replication at a stalled replication fork caused by DNA damage (Ciccia et al., 2012; Yuan et al., 2012; Weston et al., 2012). In the present study, we clarify the molecular mechanism underlying the PCNA-APIM interaction by structure-based site-directed mutagenesis. Our results provide not only a universal basis for the interaction of PCNA with both PIPM and APIM, but also significant information for the development of specific inhibitors of this interaction.

\section{Materials and methods}

\subsection{Macromolecule production}

Recombinant human PCNA was prepared using a procedure based on a previously reported protocol (Hishiki et al., 2008). Purified PCNA was concentrated to about $10 \mathrm{mg} \mathrm{ml}^{-1}$, frozen using liquid nitrogen and stored at $193 \mathrm{~K}$ until use. Information on macromolecule production for the crystallographic study is summarized in Table 1. For the interaction assay, a cDNA encoding human ZRANB3 residues 861-1079 [ZRANB3(861-1079)] was inserted into the BamHI-XhoI sites of the pGEX6P-1 vector (GE Healthcare) to express GST-fused ZRANB3(861-1079). Site-directed mutations for the interaction assay were introduced by a PCR-based mutagenesis protocol. GST-ZRANB3(861-1079) and its sitedirected mutants were expressed using Escherichia coli BL21-CodonPlus(DE3)-RIL cells (Stratagene). The cells were grown at $310 \mathrm{~K}$ to an optical density of 1.0 at $660 \mathrm{~nm}$ in TB medium, $0.1 \mathrm{~m} M$ isopropyl $\beta$-D-1-thiogalactopyranoside (IPTG) was added and the cells were cultured for a further 16$20 \mathrm{~h}$ at $288 \mathrm{~K}$. The harvested cells were suspended in a buffer composed of $20 \mathrm{~m} M$ HEPES-NaOH pH 7.4, $500 \mathrm{mM} \mathrm{NaCl}$ and lysed by sonication on ice. The cell lysate was clarified by centrifugation for $60 \mathrm{~min}$ at $48400 \mathrm{~g}$. The supernatant was applied to glutathione Sepharose 4B (GS4B) resin (GE Healthcare). The resin was washed first with a buffer composed of $20 \mathrm{~m} M$ HEPES-NaOH pH 7.4, 2.0 $M \mathrm{NaCl}$ and then with a buffer composed of $20 \mathrm{~m} M$ HEPES-NaOH, $100 \mathrm{~m} M \mathrm{NaCl}$. The GST-fused protein was eluted with a buffer composed of $50 \mathrm{~m} M$ Tris- $\mathrm{HCl} \mathrm{pH} 8.5,100 \mathrm{~m} M \mathrm{NaCl}, 50 \mathrm{~m} M$ reduced glutathione. To remove the glutathione, the eluate was dialyzed with a buffer composed of $50 \mathrm{~m} M$ Tris- $\mathrm{HCl} \mathrm{pH}$ $8.5,100 \mathrm{~m} M \mathrm{NaCl}$, frozen in liquid nitrogen and stored at $193 \mathrm{~K}$ until use.

\subsection{Crystallization}

The peptide containing the APIM from human ZRANB3 (1069-GSDITRFLVKK-1079) was commercially synthesized (Toray Research Center Inc.) and dissolved in a buffer consisting of $10 \mathrm{~m} M$ HEPES-NaOH pH 7.4, $100 \mathrm{~m} M \mathrm{NaCl}$. A tenfold molar excess of the APIM peptide was incubated with 
Table 2

Crystallization.

\begin{tabular}{ll}
\hline Method & Sitting-drop vapour diffusion \\
Plate type & 96 -well plates (VIOLAMO) \\
Temperature (K) & 293 \\
Protein concentration & $0.10 \mathrm{~m} M$ (PCNA trimer; the protein \\
& solution includes 4.0 $\mathrm{m} M$ ZRANB3 \\
& APIM) \\
Buffer composition of protein & $10 \mathrm{~m} M$ HEPES-NaOH pH 7.4, 100 m $M$ \\
$\quad$ solution & $\mathrm{NaCl}$ \\
Composition of reservoir solution & $0.2 \mathrm{M} \mathrm{NaCl}, 20 \%$ PEG 3350 \\
Volume and ratio of drop & $1: 1$ ratio protein:reservoir $(1 \mu \mathrm{l})$ \\
Volume of reservoir $(\mu \mathrm{l})$ & 40 \\
\hline
\end{tabular}

Table 3

Data collection and processing.

Values in parentheses are for the outer shell.

\begin{tabular}{ll}
\hline Diffraction source & BL-1A, Photon Factory \\
Wavelength $(\AA)$ & 1.1000 \\
Temperature $(\mathrm{K})$ & 100 \\
Detector & PILATUS 2M-F, Dectris \\
Crystal-to-detector distance $(\mathrm{mm})$ & 229.5 \\
Rotation range per image $\left({ }^{\circ}\right)$ & 1.0 \\
Total rotation range $\left({ }^{\circ}\right)$ & 90 \\
Exposure time per image (s) & 1.0 \\
Space group & $P 3_{2} 21$ \\
$a, b, c(\AA)$ & $83.68,83.68,194.93$ \\
Mosaicity $\left({ }^{\circ}\right)$ & 0.22 \\
Resolution range $(\AA)$ & $48.38-2.30(2.38-2.30)$ \\
Total No. of reflections & $168923(17020)$ \\
No. of unique reflections & $35439(3495)$ \\
Completeness $(\%)$ & $99.0(99.8)$ \\
Multiplicity & $4.8(4.9)$ \\
$\langle I / \sigma(I)\rangle$ & $13.4(2.2)$ \\
$R_{\text {meas }}$ & $0.078(0.758)$ \\
Overall $B$ factor from Wilson plot $\left(\AA^{2}\right)$ & 39.8
\end{tabular}

PCNA. Crystallization was performed by the sitting-drop vapour-diffusion method and crystals for X-ray diffraction study were obtained using a reservoir solution consisting of $0.2 \mathrm{M} \mathrm{NaCl}, 20 \%$ PEG 3350 (Table 2). Crystals were transferred into a buffer containing the reservoir solution with $20 \%$ ethylene glycol for cryoprotection.

2.3. Data collection and processing, structure solution and refinement

$\mathrm{X}$-ray diffraction data were collected on beamline BL-1A at the Photon Factory, Tsukuba, Japan using a PILATUS 2M-F pixel-array photon-counting detector (Dectris). Diffraction data were processed with $X D S$ (Kabsch, 2010) and AIMLESS (Evans \& Murshudov, 2013). The crystal structure of PCNA in complex with the ZRANB3 APIM was determined by molecular replacement with MOLREP (Vagin \& Teplyakov, 2010) using the structure of human PCNA (PDB entry 2zvm; Hishiki et al., 2009) as a probe model and was refined with Coot (Emsley et al., 2010) and REFMAC (Murshudov et al., 2011). Data-collection and refinement statistics are given in Table 3 and structure-solution and refinement statistics are given in Table 4. Atomic coordinates and structure-factor amplitudes have been deposited in the Protein Data Bank (PDB entry $5 y d 8)$.
Table 4

Structure solution and refinement.

\begin{tabular}{ll}
\hline Resolution range $(\AA)$ & $20-2.3$ \\
Completeness $(\%)$ & 98.2 \\
$\sigma$ Cutoff & None \\
No. of reflections, working set & 31833 \\
No. of reflections, test set & 1771 \\
Final $R_{\text {cryst }}$ & 0.220 \\
Final $R_{\text {free }}$ & 0.264 \\
No. of non-H atoms & \\
$\quad$ Protein & 5974 \\
$\quad$ Water & 45 \\
Total & 6019 \\
R.m.s. deviations & \\
Bonds $(\AA)$ & 0.011 \\
$\quad$ Angles $\left({ }^{\circ}\right)$ & 1.508 \\
Average $B$ factors $\left(\AA^{2}\right)$ & \\
$\quad$ Protein & 52.4 \\
$\quad$ Water & 44.8 \\
Ramachandran plot & \\
$\quad$ Most favoured $(\%)$ & 98 \\
Allowed $(\%)$ & 2 \\
\hline
\end{tabular}

\subsection{Interaction analysis by GST pull-down assay}

Competition binding for PCNA between triiodothyronine (T3), a PCNA inhibitor (Punchihewa et al., 2012), and APIM was performed using the following procedure. A mixed solution of GST-ZRANB3(861-1079) and PCNA was incubated with GS4B resin at $277 \mathrm{~K}$. T3 was then added to the resin in a 1:1 or 1:10 molar ratio relative to the PCNA monomer. After overnight immobilization, the resin was washed four times with a buffer composed of $50 \mathrm{mM}$ Tris- $\mathrm{HCl} \mathrm{pH} 8.5,0.2 \mathrm{M}$ $\mathrm{NaCl}$. Bound proteins were analyzed by SDS-PAGE with Coomassie Brilliant Blue staining. The pull-down experiment was performed three times and consistent results were obtained. Band intensities were detected and calculated using a ChemiDoc Touch imaging system (Bio-Rad). Interaction analysis between PCNA and APIM was performed with GSTZRANB3(861-1079) or its mutant proteins using a similar procedure but without $\mathrm{T} 3$. These assays were performed three times.

\section{Results}

3.1. Crystal structure of PCNA in complex with the ZRANB3 APIM

The crystal structure of human PCNA bound to a human ZRANB3 peptide carrying an APIM peptide (1069-GSDITRFLVKK-1079; the APIM consensus residues are underlined) was determined at $2.3 \AA$ resolution (Figs. $2 a$ and $2 b$ ). The bound ZRANB3 APIM peptide adopts an extended structure with a one-turn helix. The peptide is bound to the outer hydrophobic surface of the ring structure adjacent to the interdomain connector loop (IDCL) in each subunit of the PCNA trimer. The crystal structure suggests that the APIM-binding site on PCNA is similar to the PIPM-binding site (Figs. $2 c$ and $2 d$ ). Structural studies of PCNA in complex with PIPM peptides previously revealed that PIPM adopts an extended structure with one short helix, and identified two major binding pockets for PIPM on the surface of PCNA (Fig. $2 d$; 
Gulbis et al., 1996; Bruning \& Shamoo, 2004; Sakurai et al., 2005; Kontopidis et al., 2005; De Biasio et al., 2015; Wang et al., 2016). One, termed the 'Q-pocket' here, accommodates the glutamine residue positioned at P1 of PIPM (Fig. 1a) through hydrogen bonds and van der Waals contacts (Fig. 2d). Noncanonical PIPMs make a diverse interaction with the Q-pocket (Hishiki et al., 2009). The other pocket exposes a hydrophobic surface and binds hydrophobic and aromatic residues at P4, P7 and P8 of PIPM (Met147, Phe150 and Tyr151 in p21) via van der Waals contacts (Fig. 2d). These residues adopt a tripartite structure termed a 'hydrophobic plug'.

To confirm the interaction between PCNA and the ZRANB3 APIM observed in the crystal structure, and to exclude the possibility that these findings were an artifact of crystallization or the use of a peptide, competition binding for PCNA between GST-ZRANB3(861-1079) and T3 was carried out by GST pull-down assays (Fig. 3). T3 is a PCNA inhibitor that binds to the hydrophobic pocket of the PIPM-binding site (Fig. 3, left panel), thereby disturbing the interaction between p21 and PCNA (Punchihewa et al., 2012). Firstly, the interaction between GST-ZRANB3(861-1079) and PCNA was confirmed as a positive control (lane 2). Next, the addition of an equimolar amount (lane 3 ) or a tenfold molar excess (lane 4) of T3 was shown to markedly reduced the interaction of GST-ZRANB3(861-1079) and PCNA. This result definitively indicates that the binding sites of APIM and PIPM on PCNA

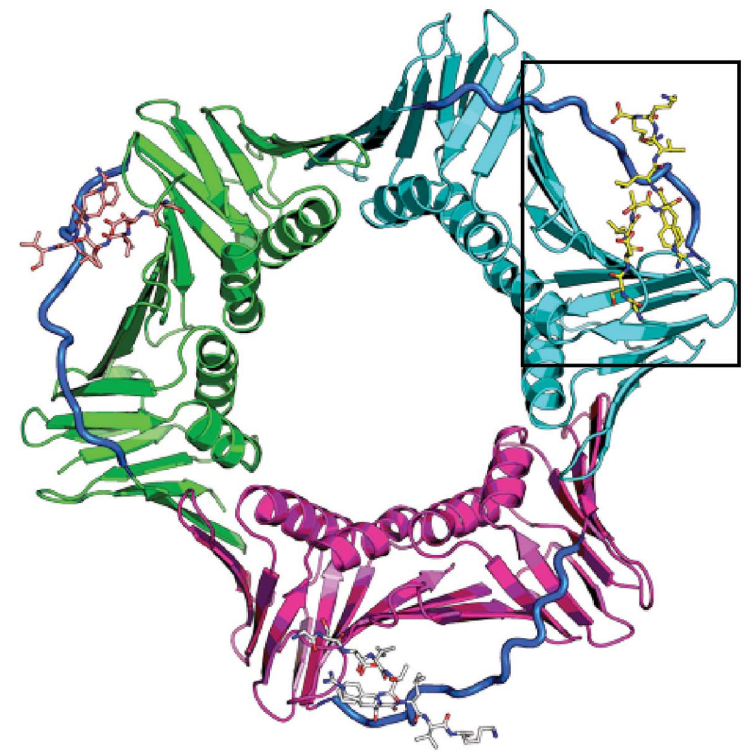

(a)

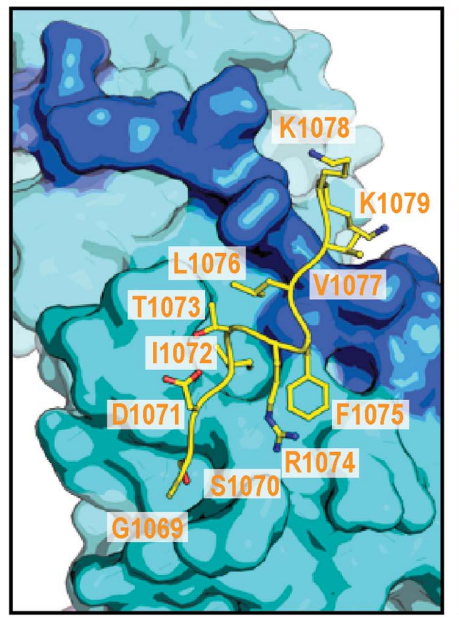

(c)

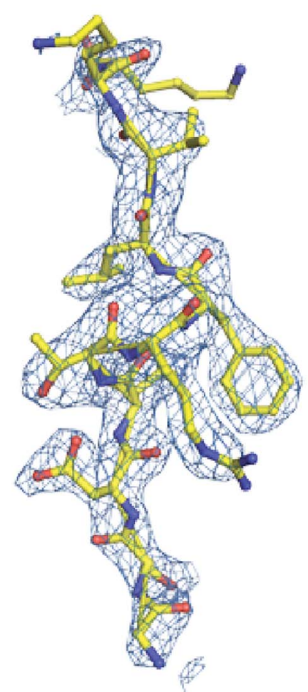

(b)
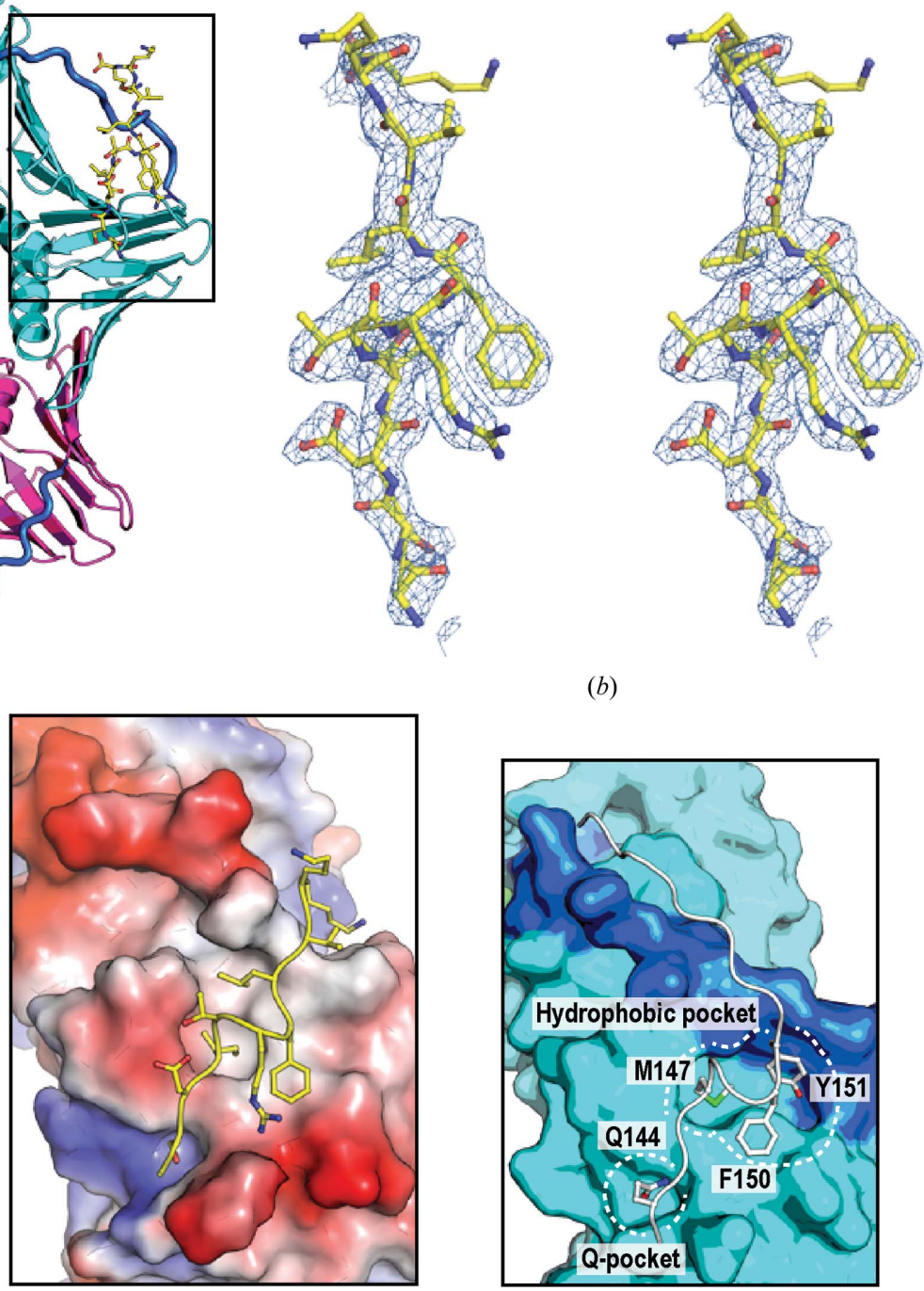

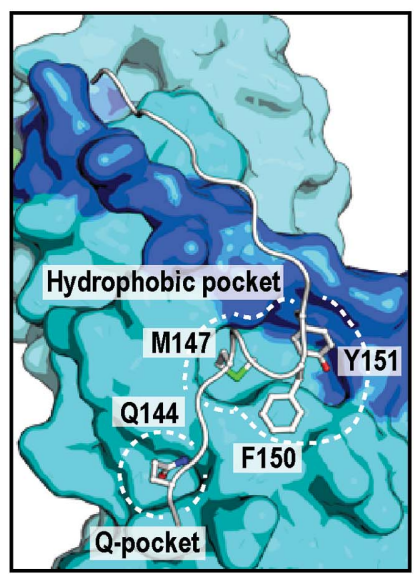

(d)

Figure 2

(a) Overall structure of PCNA with bound APIM. PCNA is shown in ribbon representation and the subunits in the PCNA trimer are shown in green, cyan and magenta. The IDCL (Leu118-Tyr133) of each PCNA subunit is shown as a thick tube coloured in blue. APIMs bound to the PCNA subunits are shown in stick representation (yellow, pink and grey). (b) Stereoview of the electron density of the APIM peptide boxed in $(a)$ as a representative (weighted $2 F_{\mathrm{o}}-F_{\mathrm{c}}$ map contoured at $1.0 \sigma$ ). (c) Binding of APIM to PCNA. Left: the molecular surface of PCNA is shown in cyan and the IDCL is highlighted in blue. APIM is shown as a yellow tube. The side chains of all residues are shown in stick representation. Right: the electrostatic potential of PCNA is mapped onto the molecular surface. Blue and red surfaces indicate positive and negative potentials, respectively. (d) Binding of the canonical PIPM of p21 to PCNA. The structure of p21 bound to PCNA is shown as a silver tube (PDB entry 1axc; Gulbis et al., 1996). Consensus residues in PIPM are shown in stick representation and are labelled. PCNA is shown as a surface representation as in the left panel of $(c)$. 


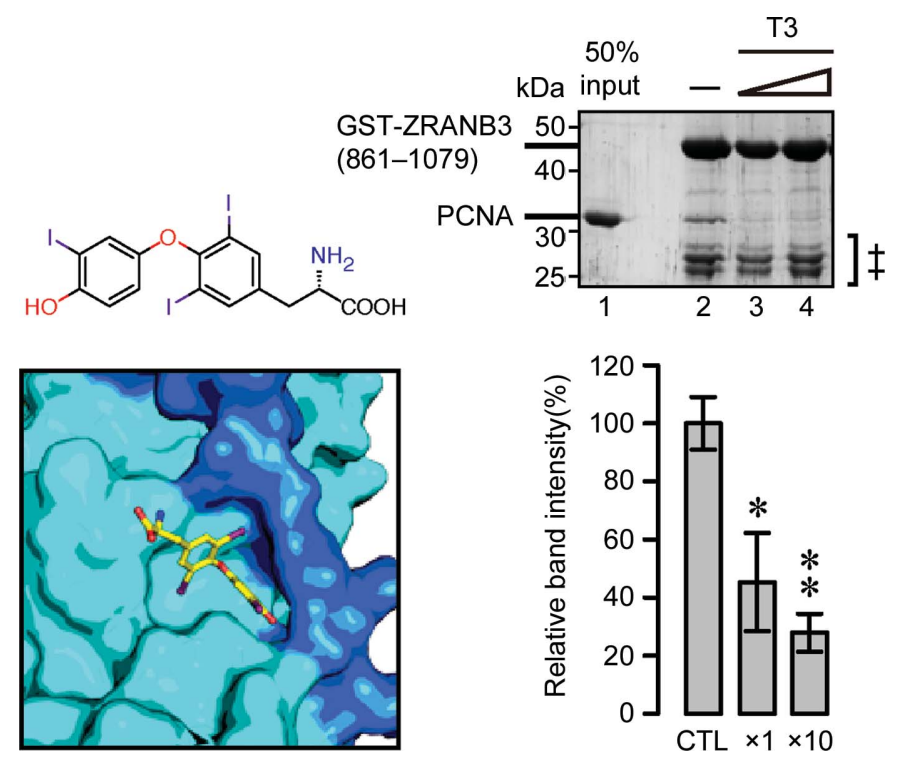

Figure 3

Competition analysis between APIM and T3 by pull-down assay. The left panel shows PCNA bound to T3 (PDB entry 3vkx; Punchihewa et al., 2012). The molecular surface of PCNA is shown in cyan and blue (IDCL) and T3 is shown in stick representation. Lane 1 contains PCNA only. Lane 2 contains GST-ZRANB3(861-1079), which includes the APIM peptide, incubated with PCNA as a control (CTL). Lanes 3 and 4 contain GST-ZRANB3(861-1079) incubated with PCNA in the presence of an equimolar amount and a tenfold molar excess of T3, respectively. The double dagger indicates the degradation products of GST-ZRANB3(8611079) that supposedly retain the GST moiety but lack the C-terminal APIM region, and are independent of the interaction between GSTZRANB3(861-1079) and PCNA. The relative band intensities of PCNA divided by the band intensities of GST-ZRANB3(861-1079) were normalized to those of CTL (lane 2), and the values obtained are presented as bar graphs with standard error (SE) bars $(n=3)$. Asterisks indicate statistically significant differences from the control (CTL) (Dunnett's test; *, $p<0.05 ; * *, p<0.01$ ). overlap. Moreover, it is reasonable to consider that the interactions observed in the present structure reflect those of the ZRANB3 protein with PCNA. This justifies the use of the ZRANB3 peptide in the present study.

The overall structure of the ZRANB3 APIM bound to PCNA seems to be similar to those of PIPMs such as the p21 PIPM (Figs. $2 c, 2 d$ and $4 a$ ). In brief, Ser1070 is bound to the Q-pocket and Ile1072, Phe1075 and Leu1076 are bound to the hydrophobic pocket. However, the structural orientation of Leu1076 in ZRANB3 differs from that of the corresponding residue positioned at P8 of the canonical PIPM (Tyr151 in p21). Instead, the orientation of Leu1076 shows similarity to that of Leu453 in Pol- $\iota$, which has a noncanonical PIPM (Figs. $4 a$ and $4 b$ ). These observations imply that the mechanism of PCNA interaction is conserved for APIM and PIPM. Details of the interactions are described in the following section.

\subsection{Structural details of the interaction between the ZRANB3 APIM and PCNA}

The ZRANB3 APIM is bound to PCNA through van der Waals and electrostatic interactions (Figs. $5 a$ and $5 b$ ). The aromatic and hydrophobic residues within the consensus APIM (Phe1075, Leu1076 and Val1077) are markedly involved in van der Waals interactions with PCNA. Phe1075 and Leu1076 within APIM, and Ile1072 preceding it, unexpectedly form a tripartite hydrophobic plug with a helical conformation, as observed for PIPM (Figs. $2 c$ and $2 d$ ). The helical conformation might be stabilized by an intramolecular hydrogen bond between the main-chain atoms of Thr1073 and Leu1076 (Fig. 5a). The hydrophobic plug is bound to the hydrophobic pocket of PCNA through van der Waals contacts

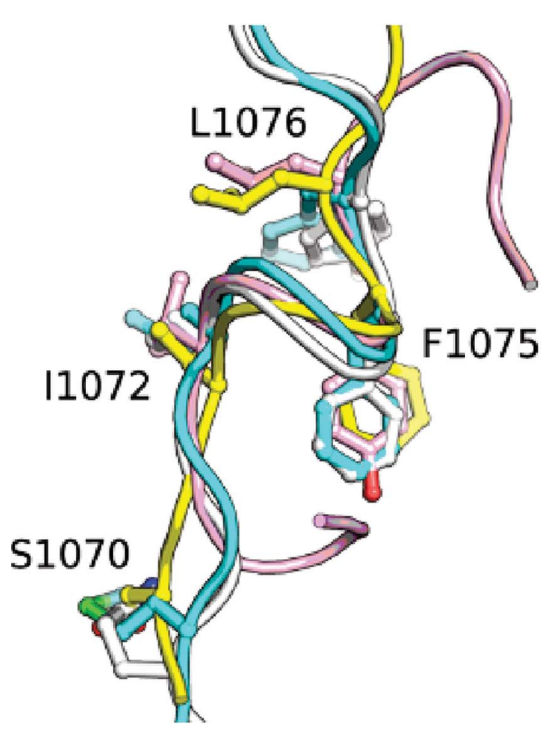

Figure 4

(a) Stereoview of the structural overlay of ZRANB3 APIM (yellow) and PIPMs: p21 (PDB entry 1axc; silver), Pol- $\iota$ (PDB entry 2zvm; pale pink) and Pol- $\eta$ (PDB entry 2zvk; pale cyan; Hishiki et al., 2009). Residues of ZRANB3 APIM are labelled. (b) Binding of the noncanonical PIPM of Pol- $\iota$ to PCNA. The structure of Pol- $\iota$ bound to PCNA is shown as a pale pink tube (PDB entry 2zvm). Residues of the 'hydrophobic plug' (Ile449, Tyr452 and Leu453) are shown in stick representation and are labelled. Residues engaged in intramolecular contacts (Lys446 and Tyr451) are also shown. PCNA is shown as a surface representation and the colours correspond to those in the left panel of Fig. 2(c). 
(Figs. $2 c$ and $5 b$ ). Except for Leu1076, the interactions of the hydrophobic plug with PCNA are remarkably similar to those of the p21 PIPM, as briefly described above. More specifically, the side chain of Leu1076 of ZRANB3 is bound to an aliphatic subsite surrounded by Met40, Leu47, Leu126, Gly127 and
Ile128 of PCNA, and is involved in an intramolecular contact with the side chain of Ile1072 of ZRANB3 (Figs. $5 a$ and $5 b$ ). The aliphatic environment of the subsite might be favoured by Leu1076; if so, the structural orientation of Leu1076 would differ from that of the corresponding residue of PIPM

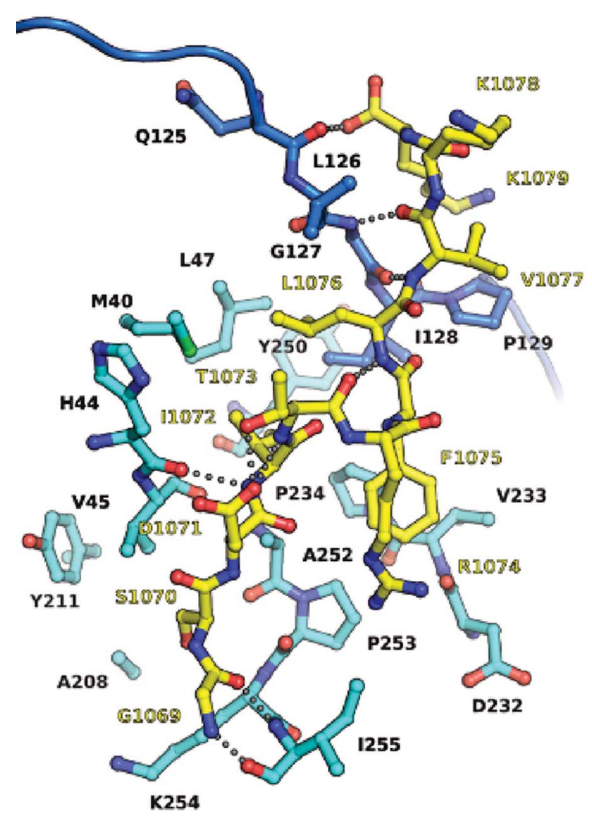

(a)

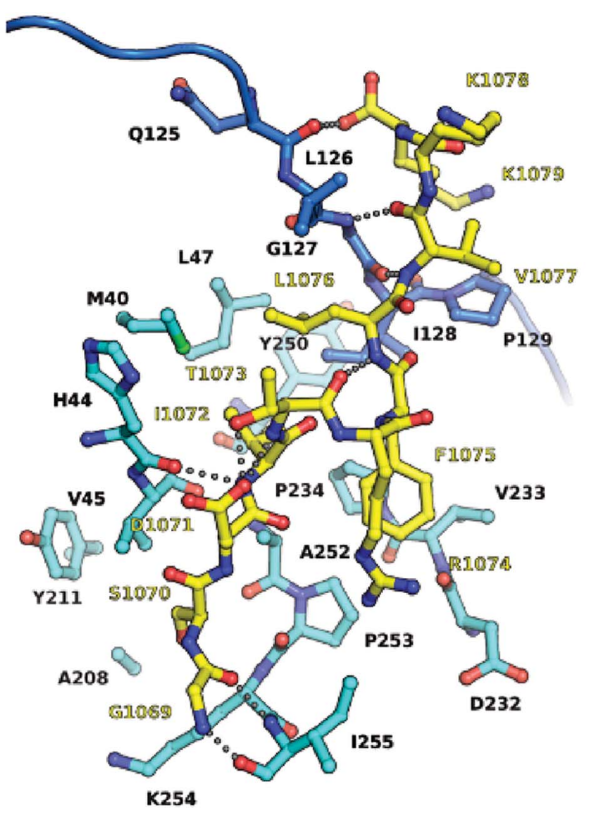

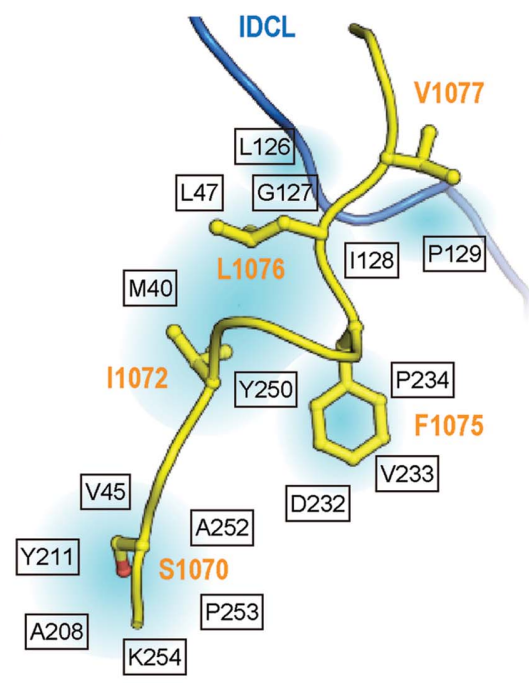

(b)

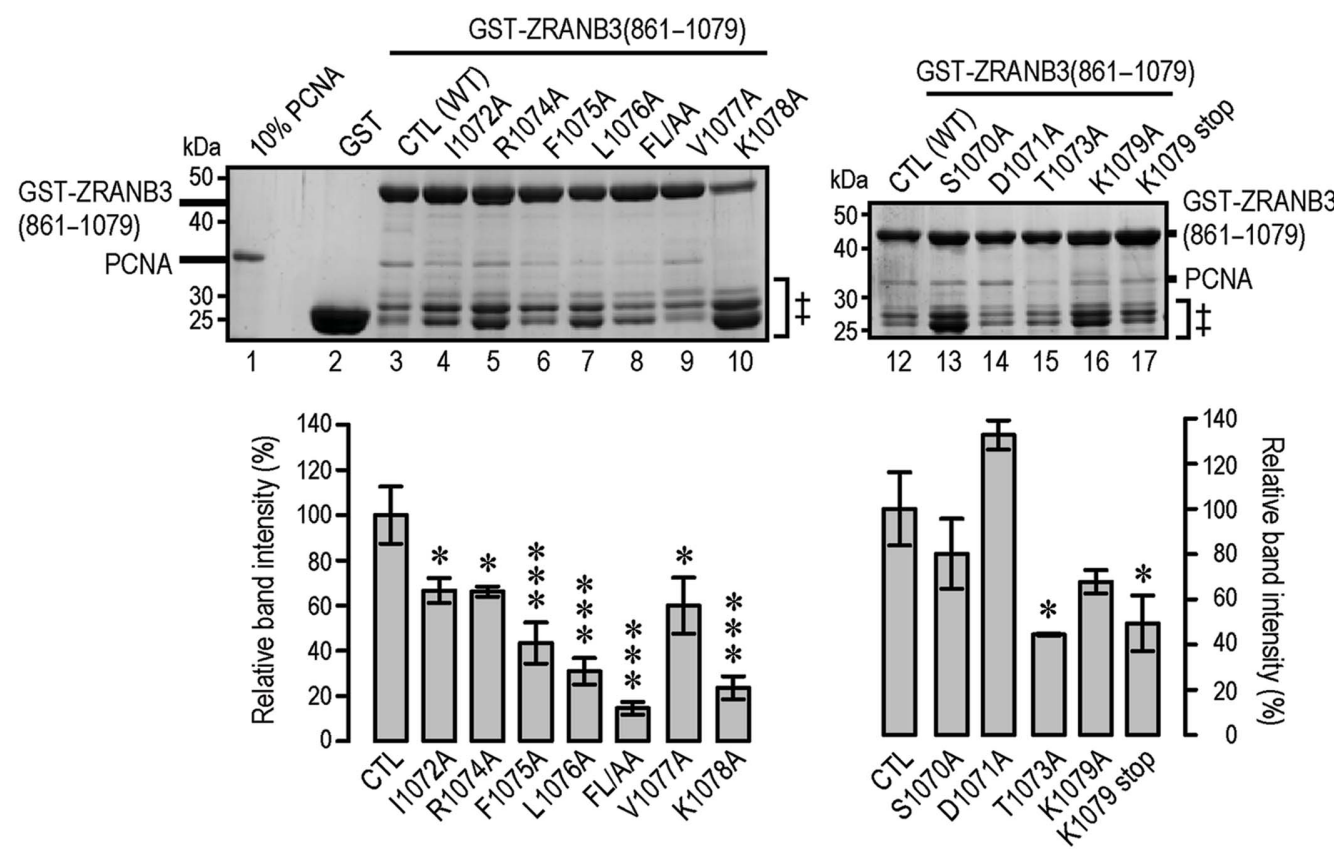

(c)

Figure 5

(a) Stereoview of detailed interactions between the ZRANB3 APIM (yellow) and PCNA (cyan). The IDCL of PCNA is shown in blue as in Fig. 2(a) and the left panel of Fig. 2(c). Electrostatic interactions are shown as grey dots. Residues of the ZRANB3 APIM and PCNA are labelled in yellow and black, respectively. (b) Schematic drawing of van der Waals interactions of the ZRANB3 APIM with PCNA. The ZRANB3 APIM and the ICDL of PCNA are shown as cartoon representations. The colours correspond to those in Fig. 2(a). Cyan bleeding indicates van der Waals interactions with the surrounding residues of PCNA indicated in boxes. (c) Interaction analysis between the ZRANB3 APIM and PCNA. The double dagger is defined in the legend of Fig. 3. Lane 1 contains PCNA only. Lane 2 contains GST and PCNA, showing no interaction between GST and PCNA. Lane 3 contains wild-type GSTZRANB3(861-1079) as a positive control (CTL). Lanes 3-17 contain GST-ZRANB3(861-1079) with single or double mutations. FL/AA indicates the F1075A/L1076A double mutant of GST-ZRANB3(861-1079). The relative band intensities of PCNA are shown as in as Fig. 3 ( $n=3$; Dunnett's test; $*, p<0.05$; ***, $p<0.001)$. 
(Fig. 4b), where the aromatic side chain of the P8 residue (Tyr151 in the p21 PIPM) is bound to a subsite surrounded by Ile128, Pro129, Gln131, Val233 and Pro234 of PCNA (Gulbis et al., 1996). Consistent with these considerations, a similar interaction is observed in the noncanonical PIPM of Pol- $\iota$, which has a leucine residue at P8 (Leu453; Figs. $1 a, 4 a$ and $4 b$; Hishiki et al., 2009). Owing to the distinctive conformation of Leu1076, the following Val1077 of ZRANB3 faces towards Pro129 of PCNA and interacts with its side chain by van der Waals contacts (Figs. $5 a$ and $5 b$ ).

The first basic residue within the consensus APIM, Arg1074, makes no contact with PCNA, but its side chain is engaged in an intramolecular interaction. The aliphatic portion of the side chain makes a van der Waals contact with the aromatic side chain of Phe1075, and the positively charged portion of Arg1074 might be partially involved in a cation $-\pi$ interaction with the aromatic side chain of Phe1075 (Fig. 5a). In contrast to Arg1074, the second basic residue, Lys1078, seems to make no obvious contact with either PCNA or other residues in the peptide because the electron density of its side chain is ambiguous, indicating that the side chain is unlikely to be involved in a rigid interaction. As described above, Ile1072 of ZRANB3 preceding the consensus APIM is also involved in the interaction with PCNA. Furthermore, the following interactions of residues outside the consensus APIM are also observed in the structure. Firstly, the side chain of Ser1070 is accommodated within the Q-pocket, the binding pocket of the conserved glutamine residue within canonical PIPMs, through van der Waals contacts (Fig. $5 b$ ). Secondly, the side chain of Asp1071 forms intramolecular hydrogen bonds to the side chain and main chain of Thr1073. In addition to these specific side-chain interactions, main-chain atoms of Gly1069, Ile1072, Val1077 and Lys1079 form hydrogen bonds to main-chain atoms of PCNA (Fig. 5a).

\subsection{Key residues in the interaction between the ZRANB3} APIM and PCNA

To pinpoint the crucial residues that interact with PCNA and the mechanism underlying the PCNA-APIM interaction, pull-down assays were performed using GST-ZRANB3(8611079) with site-directed mutations (Fig. 5c). Mutations in the residues of the hydrophobic plug (I1072A, F1075A and L1076A) significantly reduced binding to PCNA (lanes 4, 6 and 7), indicating that van der Waals contacts with the hydrophobic pocket of PCNA are essential for the interaction. In particular, Phe1075 and Leu1076 were found to be crucial for binding as F1075A, L1076A and F1075A/L1076A mutations had the great impact on the binding (lanes 6,7 and 8). A V1077A mutation led to reduced binding; thus, the van der Waals contact between Val1077 and Pro129 in the IDCL also contributes to the interaction (lane 9). Mutations of two basic residues within APIM, Arg1074 and Lys1078, to alanine also had an impact on binding (lanes 5 and 10). As described in the previous section, Arg1074 is engaged in intramolecular interactions with the aromatic side chain of Phe1075. Interestingly, mutation of Thr1073, the side chain of which is involved in an intramolecular interaction, to alanine also decreased the binding to PCNA (lane 15). These results indicate that these intramolecular interactions within the ZRANB3 APIM contribute to the interaction with PCNA.

Conversely, a D1071A mutation did not affect binding to PCNA (lane 14); rather, it seemed to confer a slight increase in binding affinity. The negative side chain of Asp1071 might cause slight electrostatic repulsion of the negative potential of PCNA (Fig. $2 c$, right). Unexpectedly from the crystal structure, a K1078A mutation largely reduced binding to PCNA, and this reduction was comparable to that caused by mutation of Phe1075 and Leu1076 to alanine (lane 10). Although the side chain of Lys1078 makes no rigid contacts in the crystal structure, it might be employed in a transient interaction with the negative potential of PCNA during the binding process (Fig. 2c, right). Mutation of Ser1070 accommodated in the Q-pocket to alanine did not affect binding to PCNA, suggesting that van der Waals contacts between Ser1070 and the Q-pocket are negligible in this complex (lane 13). In fact, the noncanonical PIPMs of Pol $\iota$ and Pol- $\kappa$ make no contact with the Q-pocket (Hishiki et al., 2009). Mutation of Lys1079, the side chain of which is also flexible in the crystal structure, to alanine had little effect on PCNA binding (lane 16), whereas truncation of the C-terminal Lys1079 had an impact on the interaction (lane 17). Consistent with this, the $\mathrm{C}$-terminus of the peptide interacts with the carbonyl $\mathrm{O}$ atom of Leu126 in the IDCL of PCNA (Fig. 5a).

Taken together, these findings indicate that all of the residues in the consensus APIM sequence (1074-RFLVK-1078) are significantly involved in binding to PCNA. In particular, Phe1075 and Leu1076 are essential for binding, indicating that van der Waals contacts with the hydrophobic pocket of PCNA are crucial for the interaction. In addition, intramolecular interactions within the peptide make a marked contribution to PCNA binding.

\section{Discussion}

The ZRANB3 APIM binds to the PIPM-binding site of PCNA in a manner similar to PIPM. Ser1070, Ile1072, Phe1075 and Leu1076 in ZRANB3 correspond to the P1, P4, P7 and P8 residues in PIPM, respectively (Fig. 1a). Among them, Ser1070 and Ile1072 are extra residues preceding the consensus APIM. Although the contribution of Ser1070 to PCNA binding is negligible, Ile1072 is important for the interaction (Fig. 5). To date, APIMs have been found in many proteins involved in DNA processes, and five APIMs, ABH2, TFIIS-L, TFII-I $\gamma$, Topo II $\alpha$ and RAD51B (Fig. 1a, upper panel), have been functionally examined (Gilljam et al., 2009). Both $\mathrm{ABH} 2$ and TFIIS-L have a hydrophobic residue preceding the consensus APIM: Met1 in ABH2 and Met1 in TFIIS-L. These hydrophobic residues might be a crucial component of the hydrophobic plug accommodated in the hydrophobic pocket of PCNA, as observed in ZRANB3. In contrast, TFII-I $\gamma$, Topo II $\alpha$ and RAD51B lack this hydrophobic residue, suggesting that the interaction of these proteins with PCNA might be weakened. The fourth residue in the consensus APIM is 
alanine in RAD51B, but is a branched aliphatic residue in the other proteins. Because mutation of Val1077 to alanine in ZRANB3 reduced the interaction with PCNA (Fig. 5c, lane 9), the methyl side chain of alanine is likely to be too small to contact Pro129 in the IDCL of PCNA (Fig. 5a). In fact, RAD51B was observed to show less prominent binding to PCNA (Gilljam et al., 2009). TFII-I $\gamma$ has four APIMs, APIM1-APIM4, which all function to interact with PCNA (Fattah et al., 2014). All four TFII-I $\gamma$ APIMs lack the hydrophobic residue corresponding to Ile1072 of ZRANB3 and instead have a basic residue (arginine or lysine); therefore, the hydrophobic plugs of these APIMs might be incomplete. This might suggest that the individual APIMs of TFII-I $\gamma$ are not able to form a stable interaction with PCNA, and may potentially explain why multiple APIMs are required to retain the interaction.

The present structure revealed striking similarity between APIM and PIPM in both their structures and their interaction with PCNA. In particular, the APIM of ZRANB3 shares marked features with the noncanonical PIPM of Pol- $\iota$ (Fig. 4). Whereas APIM has hitherto been considered to be a PCNAbinding motif that is distinct from PIPM, it is conceivable that APIMs should be classified as a major subgroup of noncanonical PIPMs. The van der Waals interactions between the aromatic and aliphatic residues of these binding motifs and the hydrophobic pocket of PCNA are basically conserved between PIPM and APIM. Therefore, PIPM and APIM might have been derived from a common ancestor of a hydrophobic plug composed of aromatic and aliphatic residues, and subsequently evolved with fine-tuning of the interaction. During the preparation of this manuscript, another crystal structure of PCNA in complex with the ZRANB3 APIM was reported (Sebesta et al., 2017). Our structure was independently determined and the observed interactions were basically consistent with the reported structure. Based on our structure, we further explored structural features of APIM by comparison with PIPM and revealed details of the mechanism underlying the PCNA-APIM interaction. Recently, the PCNA interaction has attracted much interest as a drug target for cancer therapy (Kontopidis et al., 2005; Punchihewa et al., 2012; Müller et al., 2013; Evison et al., 2014; Olaisen et al., 2015). Our results could provide a structural basis for the development of chemical compounds or peptides to inhibit PCNA interactions for cancer therapy.

\section{Acknowledgements}

We acknowledge the kind support of the beamline staff at the Photon Factory during data collection.

\section{Funding information}

The following funding is acknowledged: Japan Society for the Promotion of Science (grant Nos. 16H04755 and 25291017 to Hiroshi Hashimoto; grant Nos. 15K18491 and 17K07314 to Kodai Hara); Ministry of Education, Culture, Sports, Science and Technology (grant No. 17H06014 to Hiroshi Hashimoto); Naito Foundation (grant to Hiroshi Hashimoto); Takeda Science Foundation (grant to Hiroshi Hashimoto).

\section{References}

Bruning, J. B. \& Shamoo, Y. (2004). Structure, 12, 2209-2219.

Ciccia, A., Nimonkar, A. V., Hu, Y., Hajdú, I., Achar, Y. J., Izhar, L., Petit, S. A., Adamson, B., Yoon, J. C., Kowalczykowski, S. C., Livingston, D. M., Haracska, L. \& Elledge, S. J. (2012). Mol. Cell, 47, 396-409.

De Biasio, A., de Opakua, A. I., Mortuza, G. B., Molina, R., Cordeiro, T. N., Castillo, F., Villate, M., Merino, N., Delgado, S., Gil-Cartón, D., Luque, I., Diercks, T., Bernadó, P., Montoya, G. \& Blanco, F. J. (2015). Nature Commun. 6, 6439.

Emsley, P., Lohkamp, B., Scott, W. G. \& Cowtan, K. (2010). Acta Cryst. D66, 486-501.

Evans, P. R. \& Murshudov, G. N. (2013). Acta Cryst. D69, 1204-1214.

Evison, B. J., Actis, M. L., Wu, S. Z., Shao, Y., Heath, R. J., Yang, L. \& Fujii, N. (2014). Bioorg. Med. Chem. 22, 6333-6343.

Fattah, F. J., Hara, K., Fattah, K. R., Yang, C., Wu, N., Warrington, R., Chen, D. J., Zhou, P., Boothman, D. A. \& Yu, H. (2014). PLoS Genet. 10, e1004419.

Gilljam, K. M., Feyzi, E., Aas, P. A., Sousa, M. M., Müller, R., Vågbø, C. B., Catterall, T. C., Liabakk, N. B., Slupphaug, G., Drabløs, F., Krokan, H. E. \& Otterlei, M. (2009). J. Cell Biol. 186, 645-654.

Gulbis, J. M., Kelman, Z., Hurwitz, J., O’Donnell, M. \& Kuriyan, J. (1996). Cell, 87, 297-306.

Hishiki, A., Hashimoto, H., Hanafusa, T., Kamei, K., Ohashi, E., Shimizu, T., Ohmori, H. \& Sato, M. (2009). J. Biol. Chem. 284, 10552-10560.

Hishiki, A., Shimizu, T., Hanafusa, T., Ohmori, H., Sato, M. \& Hashimoto, H. (2008). Acta Cryst. F64, 954-956.

Kabsch, W. (2010). Acta Cryst. D66, 125-132.

Kontopidis, G., Wu, S.-Y., Zheleva, D. I., Taylor, P., McInnes, C., Lane, D. P., Fischer, P. M. \& Walkinshaw, M. D. (2005). Proc. Natl Acad. Sci. USA, 102, 1871-1876.

Moldovan, G. M., Pfander, B. \& Jentsch, S. (2007). Cell, 129, 655-679.

Müller, R., Misund, K., Holien, T., Bachke, S., Gilljam, K. M., Våtsveen, T. K., R $\varnothing$, T. B., Bellacchio, E., Sundan, A. \& Otterlei, M. (2013). PLoS One, 8, e70430.

Murshudov, G. N., Skubák, P., Lebedev, A. A., Pannu, N. S., Steiner, R. A., Nicholls, R. A., Winn, M. D., Long, F. \& Vagin, A. A. (2011). Acta Cryst. D67, 355-367.

Olaisen, C., Müller, R., Nedal, A. \& Otterlei, M. (2015). Cell. Signal. 27, 1478-1487.

Punchihewa, C., Inoue, A., Hishiki, A., Fujikawa, Y., Connelly, M., Evison, B., Shao, Y., Heath, R., Kuraoka, I., Rodrigues, P., Hashimoto, H., Kawanishi, M., Sato, M., Yagi, T. \& Fujii, N. (2012). J. Biol. Chem. 287, 14289-14300.

Sakurai, S., Kitano, K., Yamaguchi, H., Hamada, K., Okada, K., Fukuda, K., Uchida, M., Ohtsuka, E., Morioka, H. \& Hakoshima, T. (2005). EMBO J. 24, 683-693.

Sebesta, M., Cooper, C. D. O., Ariza, A., Carnie, C. J. \& Ahel, D. (2017). Nature Commun. 8, 15847.

Vagin, A. \& Teplyakov, A. (2010). Acta Cryst. D66, 22-25.

Wang, Y., Xu, M. \& Jiang, T. (2016). Biochem. Biophys. Res. Commun. 474, 264-270.

Warbrick, E. (1998). Bioessays, 20, 195-199.

Warbrick, E., Lane, D. P., Glover, D. M. \& Cox, L. S. (1995). Curr. Biol. 5, 275-282.

Weston, R., Peeters, H. \& Ahel, D. (2012). Genes Dev. 26, 1558-1572. Yuan, J., Ghosal, G. \& Chen, J. (2012). Mol. Cell, 47, 410-421. 